\title{
MULTI-BEAM FIBER OPTIC LASER SCANNING SYSTEM FOR SURFACE DEFECT RECOGNITION
}

\author{
A. Abuazza ${ }^{\mathrm{a}}$, D. Brabazon ${ }^{\mathrm{b} *}$ and M. A. El-Baradie ${ }^{\mathrm{b}}$ \\ a Physics Department Al-Fatha University, Tripoli, Libya. \\ ${ }^{\mathrm{b}}$ School of Mechanical \&Manufacturing Engineering Dublin City University, \\ Dublin 9, Ireland \\ * corresponding author - dermot.brabazon@dcu.ie
}

\begin{abstract}
Multi-beam scanning systems are being used in automated industrial manufacturing environments to determine surface defects. Recent methods of surface defect detection involve the use of fibre-optic light emitting and detection assemblies. This paper deals with the design and development of a new high-speed photo-electronic system. A line of five emitting diodes and five receiving photodiodes were used as light sources and detectors respectively. These arrays of emitting diodes and photodetectors were positioned opposite each other. Data capture was controlled and analysed by PC using LabView software. A newly developed technique of using an angled array of fibres allows an adjustable resolution to be obtained with the system, with a maximum system resolution of approximately $100 \mu \mathrm{m}$ (the diameter of the collecting fibre core). This system was successfully used to measure various materials surface profile, surface roughness, thickness, and reflectivity. The advantages of this new system may be seen as lower cost, less bulky, greater resolution and flexibility.
\end{abstract}

KEYWORDS: Laser scanning, resolution, surface defects, fibre arrays

\section{INTRODUCTION}

Over the last three decades the attention which has been given to laser technology has increased. The light beam from a laser is monochromatic, coherenent and highly directed [1]. These properties have motivated a growing list of laser applications in the fields of measurement and inspection. The major advantages of laser scanning systems are listed as [2]: higher resolution, faster scanning speed and high contrast image acquisition. The laser scanning inspection system using triangulation technology is one of the most common and useful methods for 2-D and 3-D profiling where accurate repeatable height measurements are required [3]. Laser scanning has been successfully implemented in the inspection of widely varied material surfaces. Continuous on-line inspection of moving sheet is one of the most active fields of optical inspection [4]. Examples of sheet materials for which optical inspection systems have been reported include paper webs, textile fabric, glass material, hot slabs and cold-rolled metal strip [5]. These systems are essential tools for the implementation of modern statistical process control procedures. Non-contact methods of measuring thickness and distance with laser sensors have already been widely reported in the literature [6-9]. Very high orders of accuracy in the measurement of lengths at close ranges of up to several meters are achieved by interferometric methods $[10,11]$. These methods are however mostly too complicated to be practical for application in production [12]. An intensity-based sensor requires a much simpler optical system, and therefore, can be made very small. The working 
principle of these sensors is based on the correlation between the detected intensity of the reflected light and the average roughness of the surface. The essential problem with intensity-based sensors is that the detected intensity is strongly dependent on the gap distance between the sensor and the surface and on the surface reflectivity. The intensity of the detected light depends upon how far the reflecting surface is from the fibre optic sensor [13]. Light scattering of a test surface may be a changed by different microstructures encountered [14]. However, a sudden change in the light intensity would occur when the incident light beam encounters a defect [15].

Most previous research efforts in this area have been focused on the development of the path planning of a commercial laser scanning system [16]. Laser scanning systems have been successfully used in the inspection of widely varied material surfaces, from metals of all types to paper, glass, plastics, films, textiles, as well as magnetic and optical discs [15]. A laser scanner consists of two parts: an illumination part and an imaging part [17]. One of the most effective sensor devices currently available is the fibre optic sensor [18]. The response of an optical fibre can be affected by very small change in fibre geometry caused by conditions such as elongation, bending, or twisting [19]. These changes in response can be used to detect strains in different materials. However industrially photoelectric displacement sensing is the most common application of fibre-optic sensing [20].

Recent methods of surface defect detection involve the use of fibre-optic light emitting and detection assemblies. A newly developed technique of using an angled array of fibres allows an adjustable resolution to be obtained with the system. This system was successfully used to measure various materials surface profile, surface roughness, thickness, and reflectivity.

\section{Experimental set-up}

The main system-level components of the fibre optic sensor used in this work were the light emitter, the photo detector, the fibre waveguides, data acquisition and analysis, using LabView software. Each component plays a vital role in the quality of transmission. Careful decisions, based on system requirements, must be made for each component of the system if high-quality sensing is to be achieved. This system configuration, control and analysis, mechanical design, and resolution are discussed below.

\subsection{System configuration}

The effective application of a fibre optic system requires consideration of the entire system: the transmitter (laser diode with 1300nm), traveling signal (Multimode fibre optics, length, characteristics, and connectors), receiving detector (PIN photodiode response from $900 \mathrm{~nm}$ to $1700 \mathrm{~nm}$, preamplifier).

Figure 1 shows the structure of the fibre optic sensor, the sensor was operated using four and five $1300 \mathrm{~nm}$ multimode laser diodes. The light source with a 1300nm wavelength was chosen in order to reduce surface scattering and maximise the proportion of optical radiation that reflects specularly. 


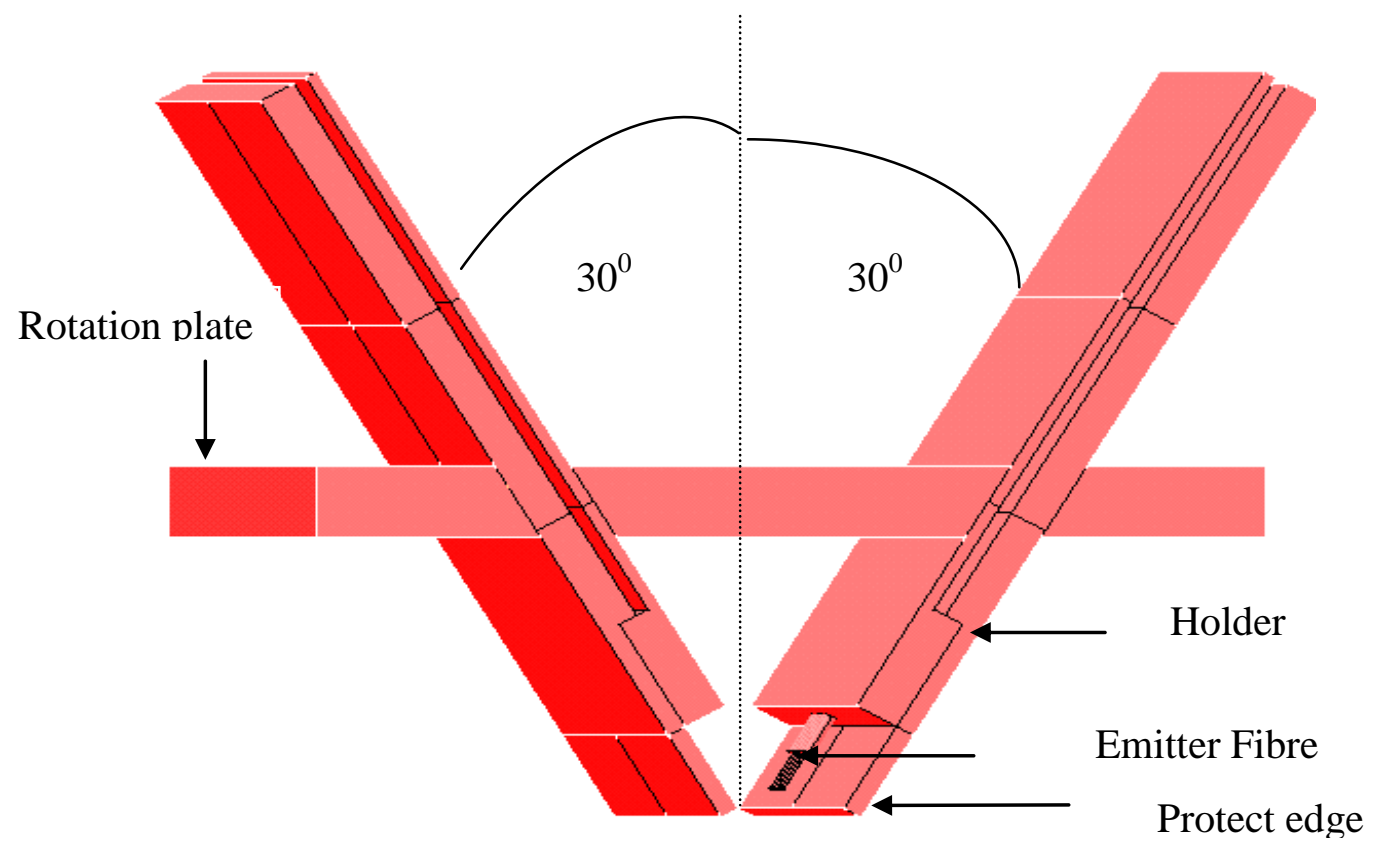

Figure 1 Configuration of fibre optics transmission system.

\subsection{LabView based data acquisition and data analysis system}

A flow chart depicting the programmes developed for data acquisition in LabView is shown in Figure 2. This system is more directly implemented when the cut-off voltage is applied as a part of measurement. Figure 3 shows the flow chart for the program that generates a surface map. The voltage level at each point $(\mathrm{x}, \mathrm{y}), \mathrm{P}_{\mathrm{xy}}$ is compared to the cut-off voltage, $\mathrm{V}_{\mathrm{c}}$. If it is less than $\mathrm{V}_{\mathrm{c}}$. the point $(\mathrm{x}, \mathrm{y})$ is added to the surface map, if it exceeds $V_{c}$ it is ignored. In this way the surface map displays the areas of the surface that are below the cut-off level. Other X-Y plots representing the light displacements against output signals (voltages) can also be displayed.
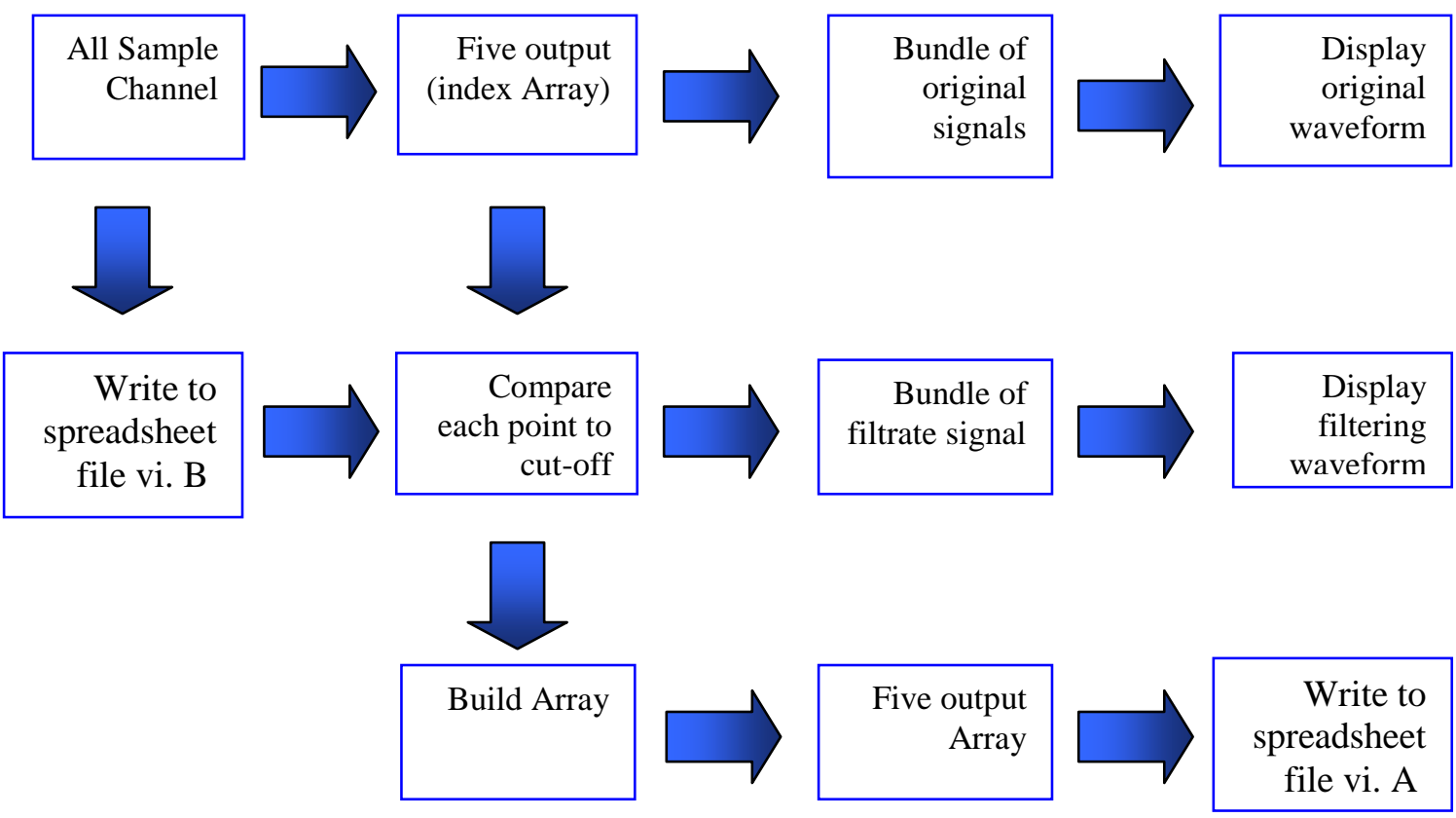

Figure 2 Surface defect sensor data acquisition programme which displays and logs the captured data. 


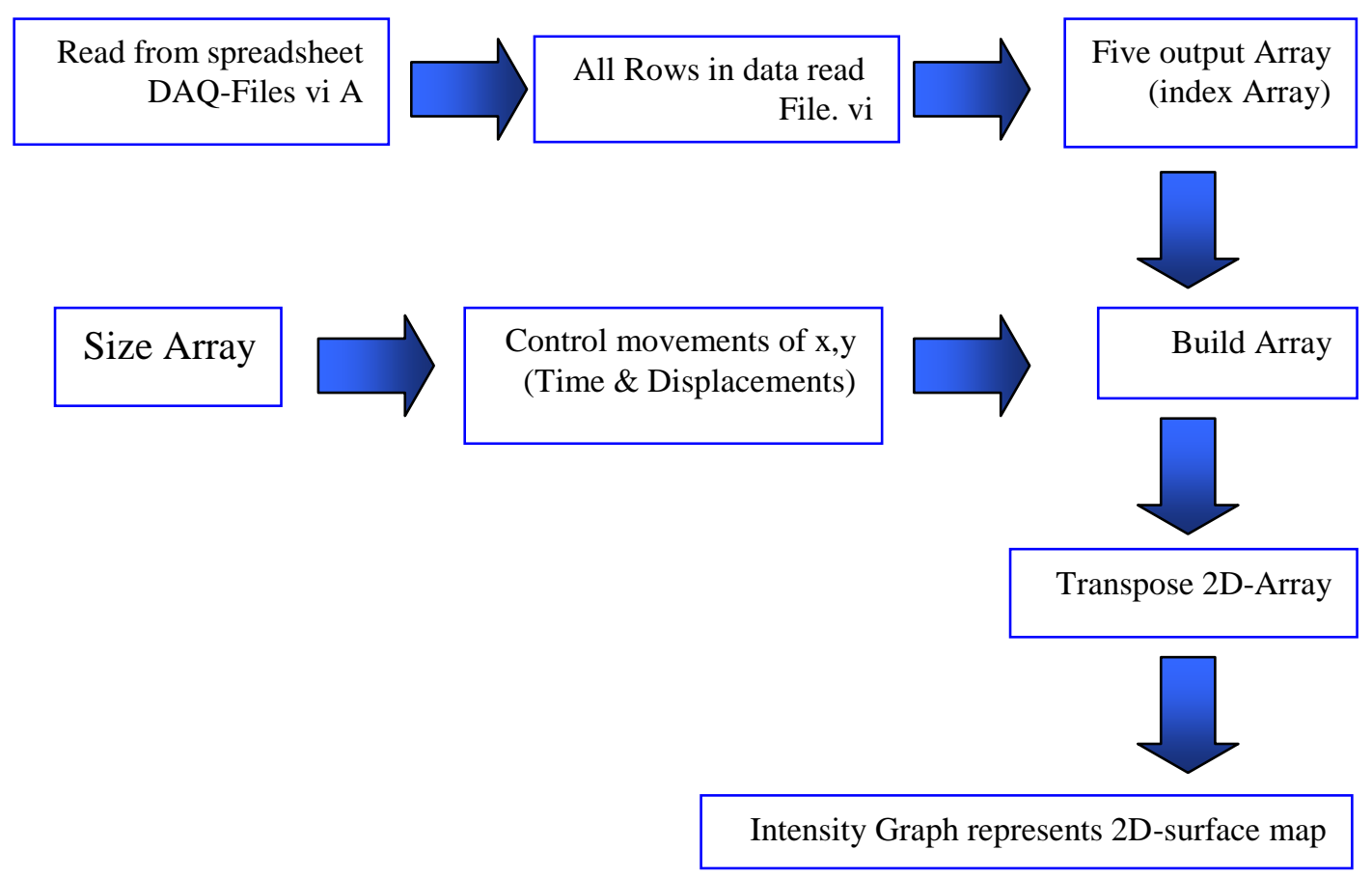

Figure 3 Surface defect surface map generation.

\subsection{Mechanical design}

The design consisted of two stepped aluminum plates. One of the plates was drilled with five holes to accommodate the fibres. The plates of the holder were fixed together with six metal screws. The fibre optic holder was designed to protect the end face of the fibre optic from any damage as shown in Figure 4.

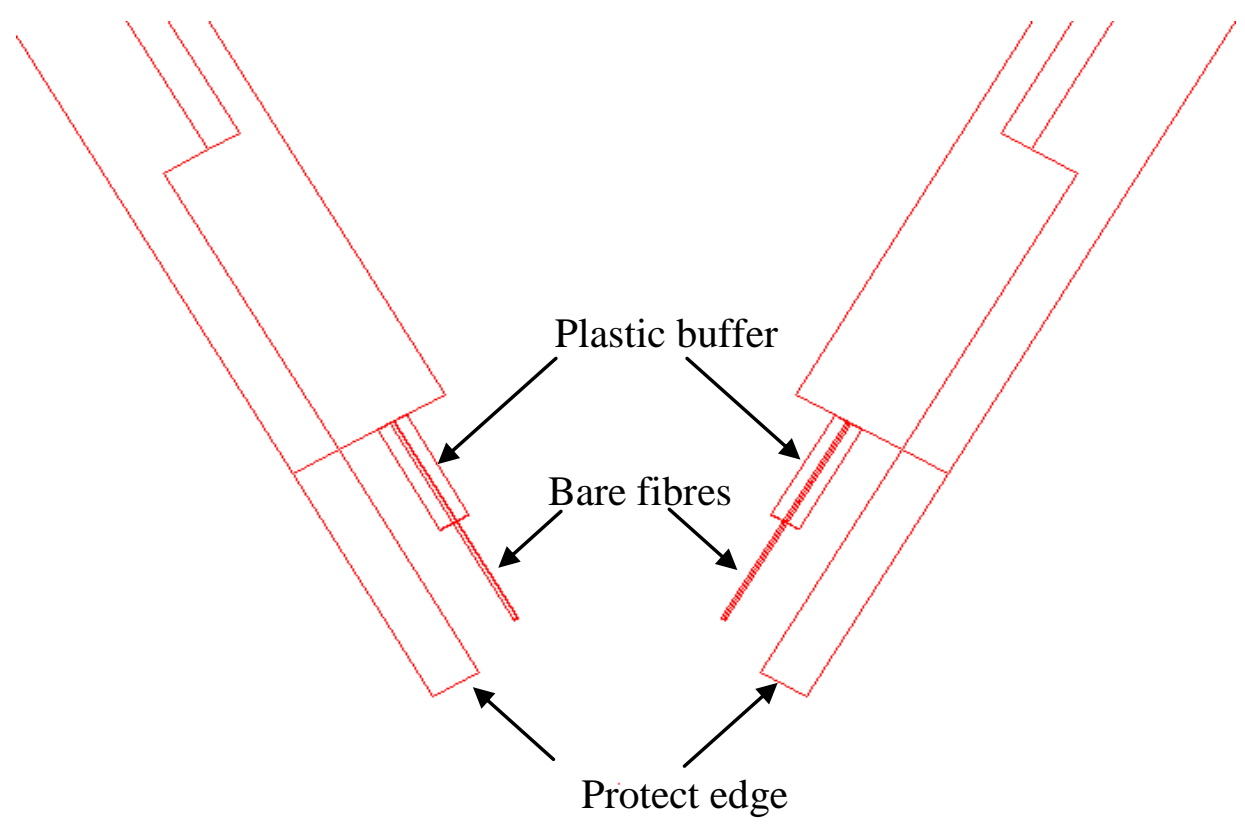

Figure 4 Close up of fibre optic holder.

\subsection{Resolution}

This section describes a technique, developed by the authors, to achieve high resolution for the present system. The novel method used four degrees of freedom. Figure 5 shows a side view of the system. Metal screws were used to fix the fibres beside each other to achieve a fixed distance between the fibres $(0.91 \mathrm{~mm})$. The novel method, used to reach high resolution, is dependent on the displacement projection of 
the spots perpendicular to the scan direction (x-axis, in Figure 6). Figure 6 (a) shows that the spots are elliptical due to the incidence angle of the light on the surface. It also shows the total distance between the spots, and the distance between any two spots of the set in the $x-y$ plane. Two diameters of the fibre spot depending on fibre, small diameter $(\mathrm{d}=62.50 \mu \mathrm{m})$ and large diameter $(\mathrm{D}=72.12 \mu \mathrm{m})$ were used.

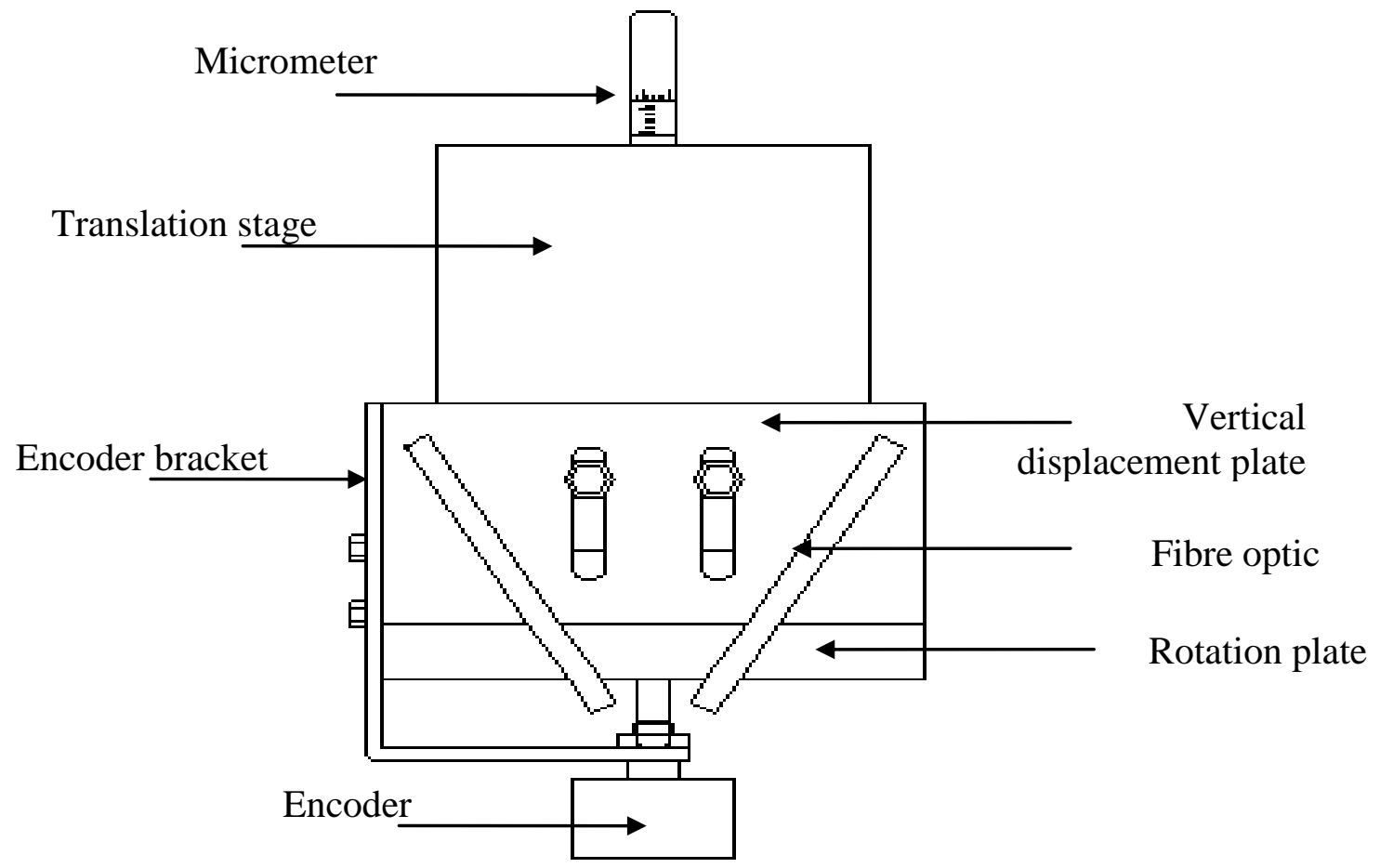

Figure 5 Side view of system

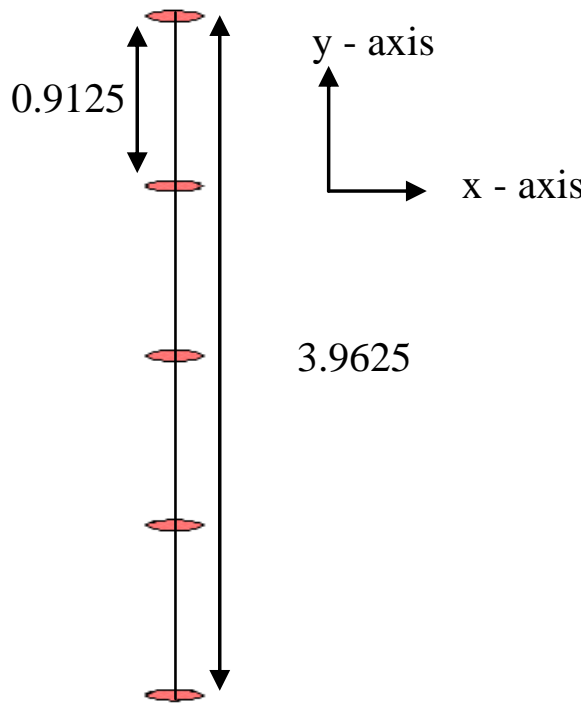

(a)

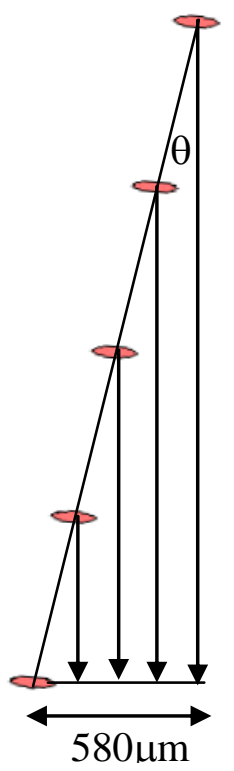

(b)

Scan direction

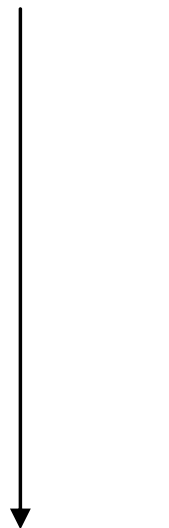

Figure 6 System resolution with (a) theoretical projection of scan at scan angle of $\theta=0^{0}$ and (b) theoretical projection of scan at scan angle of $\theta \approx 8^{0}$ 
The rotation plate was rotated in $\mathrm{x}-\mathrm{y}$ plane by angle $\theta$ as shown in Figure 6 . The angle should be $\theta \approx 8^{0}$ to achieve a projection distance $580 \mu \mathrm{m}$. The direction of the scan is in the $y$-direction on the $x-y$ plane. The experimental projections were recorded as the first and last signal drops when the five fibres passed a vertical fall in the specimen surface, which was perpendicular to the scan direction.

For the results an encoder was used to control the angle of the rotation plate. This rotary sensor was positioned between the translation stages and the rotation plate and was connected to the digital and counter input channels on the data acquisition card. A liner-guide motor simulated an on line production environment by moving the sample beneath the newly developed inspection sensor.

\section{Results and discussion}

Results from this system at variable resolution and for various materials (such as aluminum plate, transparent polycarbonate plate, brass and tufnol plate) are presented. Several materials were tested for the reflected signal intensity detected upon vertical displacement. These results indicated that all metals are very good reflectors within the infrared wavelength. The system was optimised for each material to produce the highest intensity in reflected signals. Figure 7 shows the profile of the reflected signals from the aluminum sample. Small differences in the reflected signals were caused by small misalignment of the fibres and the different points scanned on the surface.

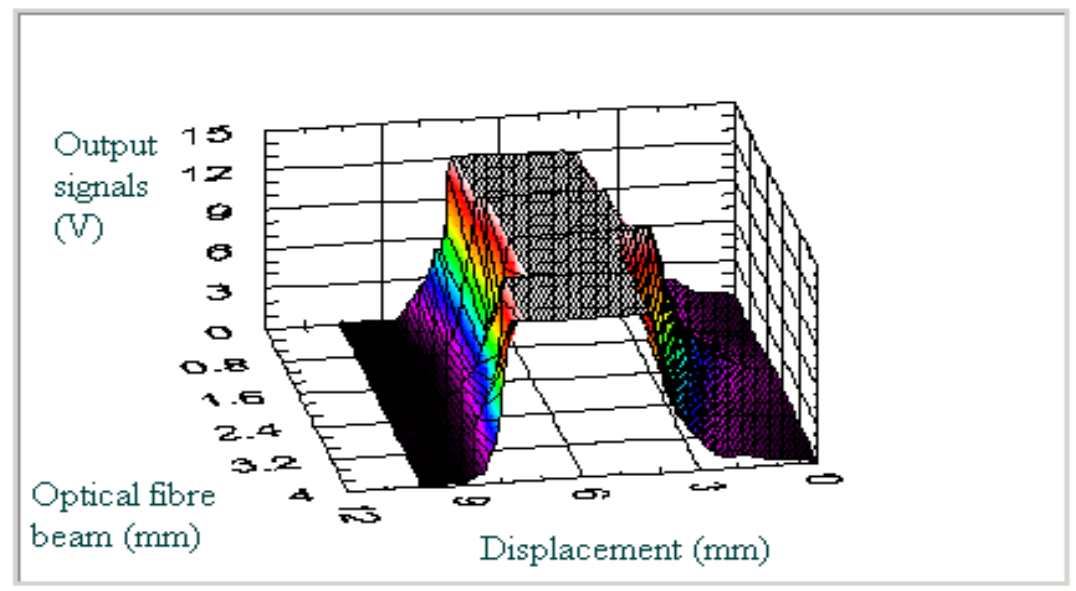

Figure 7 Profile of the reflected signals from the aluminium surface upon vertical displacement of the fibres from the surface.

The transparent polycarbonate showed a quite different than that from the metal surface. The transparent surface allowed light to pass through it diffusely. The laser spots where reflected off the top and bottom surfaces, so that two sets of signal peaks were detected. This system could therefore be used to measure the thickness of transparent material. Figure 8 shows the profile of the reflected signals for five fibres. 


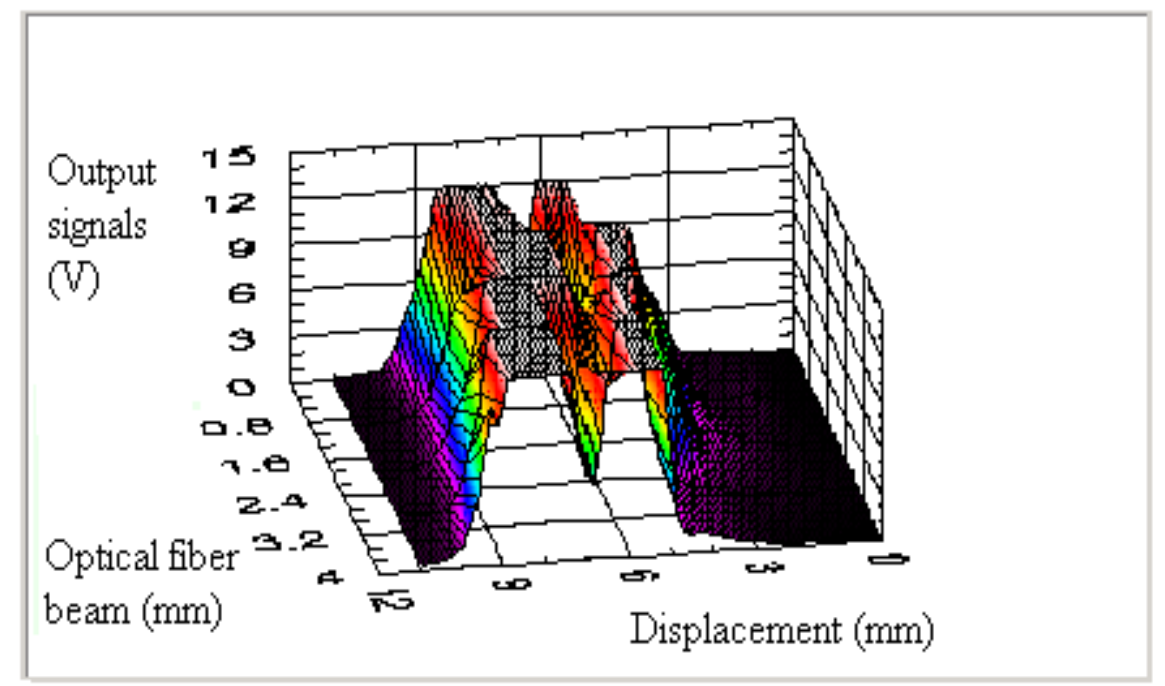

Figure 8 Profile of the reflected signals from the transparent polycarbonate surface.

The results for the diffuse tufnol material (an epoxy glass laminate with extremely high mechanical and electrical strength) are presented in Figure 9. The results show that the incident signals were significantly effected by how far the fibre ends were from the surface for this material surface. This was due to the diffuseness of the surface. Metal materials have a higher reflectivity, which allowed the system to more easily detect the reflected signals with metal samples.

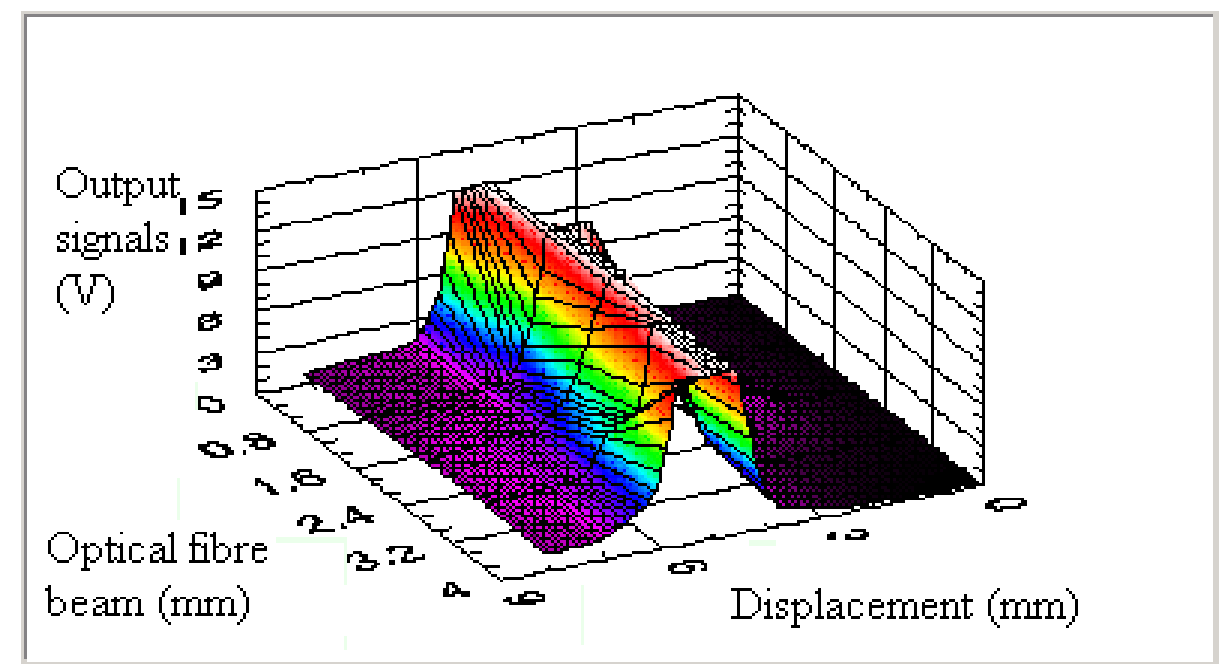

Figure 9 Profile of the reflected signals from the tufnol surface.

\subsection{Surface profile measurements}

The optimum fibre end height was set as per the results of the previous section. With the vertical displacement of the fibre ends from the surface set optimally for each material, the surface was then passed beneath the sensors in order to reconstruct the surface profile. Five beams passing over the surface enabled a notch on the aluminium 
plate to be scanned. A profile of the reflected signals from the notched surface is shown representing a irregular shaped defect, in Figure 10. The variation of the signals through the notch is due to the irregular shape of the notch.

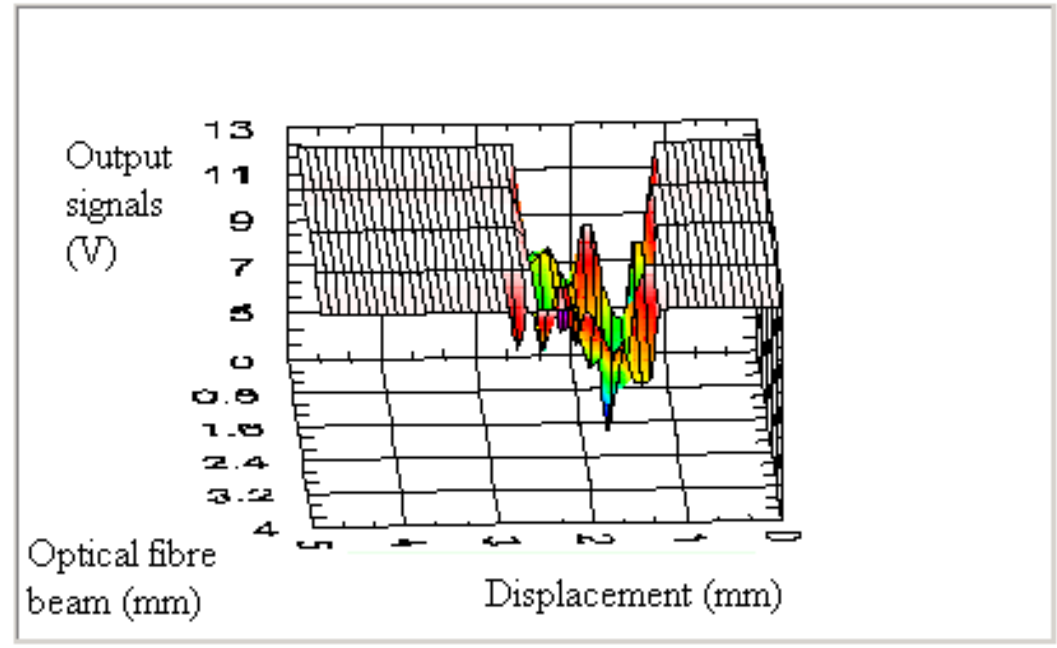

Figure 10 Profile produced from a notched aluminium surface scan.

Figure 11 shows the scan of the transparent polycarbonate notched surface. Two notches, side by side, were scanned for this measurement. The signals dropped down and were reflected again from the island between the notches before falling again as the second notch was passed.

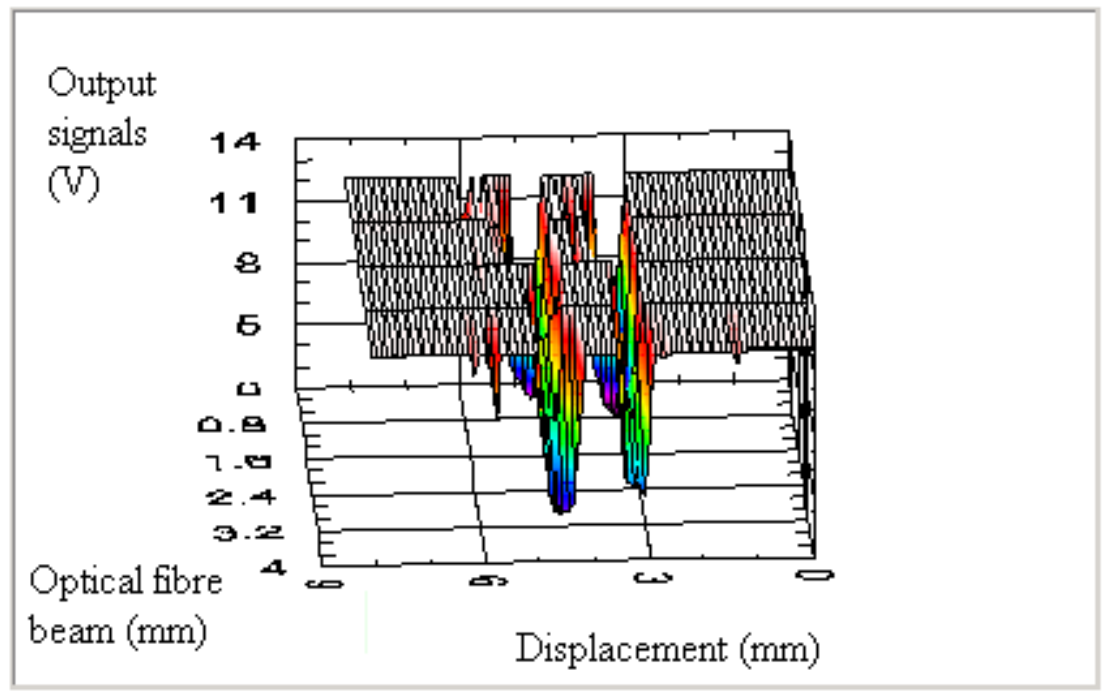

Figure 11 Profile of notch surface scan.

The diffuse tufnol surface also produced reasonable results for the surface notch measurement. Much more scatter was however detected with this material due to its diffuseness. Figure 12 represents tufnol the notched surface. 


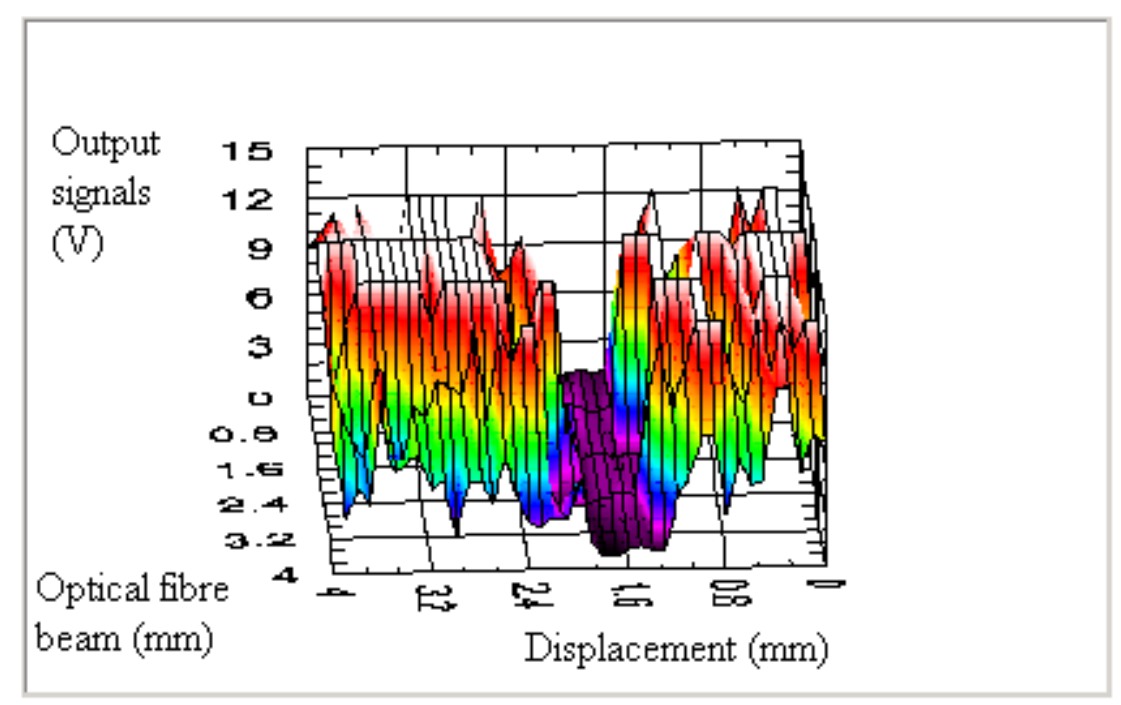

Figure 12 Profile produced from a notched tufnol surface scan.

\section{Conclusions}

In this paper, the design construction and operation of fibre optic laser scanning inspection system for surface defect was presented. The five fibres used covered a distance of up to $3.96 \mathrm{~mm}$. The last results section presented reconstructed surfaces from simulated on line inspection using a liner guide motor to move the samples under the sensor at a speed of $1.91 \mathrm{~mm} / \mathrm{s}$. A novel technique that was presented was used to adjust the resolution of the system to obtain higher accuracy results.

The resolution of the system was made adjustable by mounting the fibre holder on an adjustable rotation stage. A smallest scan line of $580 \mu \mathrm{m}$ with a defect resolution capability of $72.12 \mu \mathrm{m}$ was obtained. The experimentally obtained results from several materials shows the system's ability to recognise defects. The achieved results show that even though this system is capable of 2-D scanning it may also be operated as a limited 3-D vision inspection system. The fibre optic laser scanning system, which has been discussed in this paper offers an effective means of highly accurate measurements, high resolution, and flexibility to capture the output signal reliably.

\section{REFERENCE}

[1] H brueggemann, Low cost laser scanner, International levis design conference, 1985, SPIE vol. 554, pp457-462.

[2] S. Sullivan, Acquiring image laser scanning, 1986, Lincoln Laser Company, Phoenix Arizona.

[3] A. Abuazza, M.A. El-Baradie, Laser-scanning inspection system, an overview, AMPT, 99 and IMC 16 Conf., 1999, Dublin City University, Ireland, pp 19391945.

[4] P. Cielo, On line optical sensor for industrial inspection, 1990, In- process optical measurement and industrial methods, vol. 1266, pp. 22-38.

[5] P. Cielo, Optical techniques for industrial inspection, 1988, Academic Press, Boston.

[6] R. Pirlet, R. Franessen, V. Laforge, and F. Surleau, Slab thickness identification with the 'Optimac', 1976, CRM 46, no. 3, pp 29-34.

[7] J. Kerr, A laser thickness monitor, 1969, J. of IEEE, no. 5, part 6, pp 338-339. 
[8] V. Bodlaj, E. Klement, Remote measurement of distance and thickness using a deflected laser beam, 1976, Appl. Opt., no. 15N, part6, pp. 1432-1436.

[9] D. Rosenberger, Laser sensors for industry, 1972, Alta Frequenza, no. 41, pp. 771-779.

[10] D. Whitton, A laser technique for precise measurement, 1972, Elect. \& Power 2, pp 46-48.

[11] W. Rowley, Interferometer measurement of light and distance, 1972, Alta Freqenza 41, pp. 887-896.

[12] V. Bodlaj, Industrial application of laser, 1984, John Wiley \& Sons Ltd.

[13] G. Butler, G. Gregoriou, A novel noncontact sensor for surface topography measurement using a fibre optic principle, 1992, Sensors Actuators, vol. 31, pp 68-74.

[14] J. Stover, Optical scattering measurement and analysis, 1990, McGraw-Hill, New-York.

[15] C. Quan, S.H. Wang, Inspection of micro-crack on solderball surface using laser scattering method, 2000, Optical Communication, no. 183, pp 19-27.

[16] F. His-Yung, L. Yixin, X. Fengfeng, Analysis of digitizing errors of a laser scanning system, 2001, Precision Engineering, no. 25, pp 185-191.

[17] J. Luxon, and D.E. Parker, Industrial lasers and their pplications, 985, Prentice Hall Inc., New Jersey.

[18] E. Udd, Fibre optic smart structures, 1995, John Wiley and Sons, New York.

[19] A. Timothy and A. Hojjat, Monitoring the behaviour of steel structures using distribute optical fibre sensors, 2000, Elsevier, vol. 53. pp. 267-281.

[20] A. Anon, Laser inspection system, 1988, Optical Information Systems, vol. 8, no. 5, pp. 226-229. 